\title{
Inflammation resolution: a dual-pronged approach to averting cytokine storms in COVID-19?
}

\author{
Dipak Panigrahy ${ }^{1,2} \cdot$ Molly M. Gilligan ${ }^{1,2} \cdot$ Sui Huang ${ }^{3}$. Allison Gartung ${ }^{1,2} \cdot$ Irene Cortés-Puch $^{4,5}$ - Patricia J. Sime ${ }^{6}$. \\ Richard P. Phipps ${ }^{7}$. Charles N. Serhan ${ }^{8} \cdot$ Bruce D. Hammock $^{9}$
}

Published online: 8 May 2020

(C) Springer Science+Business Media, LLC, part of Springer Nature 2020

\begin{abstract}
Severe coronavirus disease (COVID-19) is characterized by pulmonary hyper-inflammation and potentially life-threatening "cytokine storms". Controlling the local and systemic inflammatory response in COVID-19 may be as important as anti-viral therapies. Endogenous lipid autacoid mediators, referred to as eicosanoids, play a critical role in the induction of inflammation and pro-inflammatory cytokine production. SARS-CoV-2 may trigger a cell death ("debris")-induced "eicosanoid storm", including prostaglandins and leukotrienes, which in turn initiates a robust inflammatory response. A paradigm shift is emerging in our understanding of the resolution of inflammation as an active biochemical process with the discovery of novel endogenous specialized pro-resolving lipid autacoid mediators (SPMs), such as resolvins. Resolvins and other SPMs stimulate macrophagemediated clearance of debris and counter pro-inflammatory cytokine production, a process called inflammation resolution. SPMs and their lipid precursors exhibit anti-viral activity at nanogram doses in the setting of influenza without being immunosuppressive. SPMs also promote anti-viral B cell antibodies and lymphocyte activity, highlighting their potential use in the treatment of COVID-19. Soluble epoxide hydrolase (sEH) inhibitors stabilize arachidonic acid-derived epoxyeicosatrienoic acids (EETs), which also stimulate inflammation resolution by promoting the production of pro-resolution mediators, activating anti-inflammatory processes, and preventing the cytokine storm. Both resolvins and EETs also attenuate pathological thrombosis and promote clot removal, which is emerging as a key pathology of COVID-19 infection. Thus, both SPMs and sEH inhibitors may promote the resolution of inflammation in COVID-19, thereby reducing acute respiratory distress syndrome (ARDS) and other life-threatening complications associated with robust viral-induced inflammation. While most COVID-19 clinical trials focus on "anti-viral" and "anti-inflammatory" strategies, stimulating inflammation resolution is a novel host-centric therapeutic avenue. Importantly, SPMs and sEH inhibitors are currently in clinical trials for other inflammatory diseases and could be rapidly translated for the management of COVID-19 via debris clearance and inflammatory cytokine suppression. Here, we discuss using pro-resolution mediators as a potential complement to current anti-viral strategies for COVID-19.
\end{abstract}

Keywords COVID-19 $\cdot$ SARS-CoV-2 $\cdot$ Cytokine storms $\cdot$ Inflammation resolution $\cdot$ Eicosanoid storm

Dipak Panigrahy and Molly M. Gilligan contributed equally to this work.

Dipak Panigrahy

dpanigra@bidmc.harvard.edu

Charles N. Serhan

cserhan@bwh.harvard.edu

$\triangle$ Bruce D. Hammock

bdhammock@ucdavis.edu

1 Center for Vascular Biology Research, Beth Israel Deaconess Medical Center, Harvard Medical School, Boston, MA 02215, USA

2 Department of Pathology, Beth Israel Deaconess Medical Center, Harvard Medical School, Boston, MA 02215, USA

3 Institute for Systems Biology, Seattle, WA 98109, USA
4 Division of Pulmonary, Critical Care and Sleep Medicine, University of California Davis Medical Center, Sacramento, CA 95817, USA

5 EicOsis Human Health, Davis, CA 95616, USA

6 Division of Pulmonary and Critical Care Medicine, Virginia Commonwealth University, Richmond, VA 23298, USA

7 Independent scholar, Richmond, VA 23233, USA

8 Center for Experimental Therapeutics and Reperfusion Injury, Department of Anesthesiology, Perioperative and Pain Medicine, Brigham and Women's Hospital, Harvard Medical School, Boston, MA 02115, USA

9 Department of Entomology and Nematology, and UCD Comprehensive Cancer Center, University of California, Davis, Davis, CA 95616, USA 
Severe coronavirus disease (COVID-19) caused by the SARS-CoV-2 virus is frequently characterized by pulmonary inflammation [1]. Life-threatening "cytokine storms" involving the release of pro-inflammatory cytokines (e.g., TNF- $\alpha$, IL-6, IL-1, IL-8, and MCP-1) may contribute to the rapid systemic organ failure observed in select critically ill COVID-19 patients [1]. However, this storm is not a selflimiting, singular event. SARS-CoV-2 causes massive cell death and cellular debris that activates inflammasomes [2], which in turn trigger a macrophage-derived "eicosanoid storm", a surge of pro-inflammatory bioactive lipid mediators, such as prostaglandins and leukotrienes, that fuels local inflammation [3-5]. A paradigm shift in the inflammation field is that the resolution of inflammation is an active biochemical process [5], implying that hyper-inflammation may result from a deficit in resolution.

In contrast to classic anti-inflammatory agents, endogenous pro-resolution lipids can terminate the inflammatory response by promoting the clearance of cellular debris. Specialized proresolving mediators (SPMs), including resolvins, lipoxins, and protectins, are bioactive lipid autacoids that mediate

\section{COVID-19 INJURY AND INFLAMMATION}

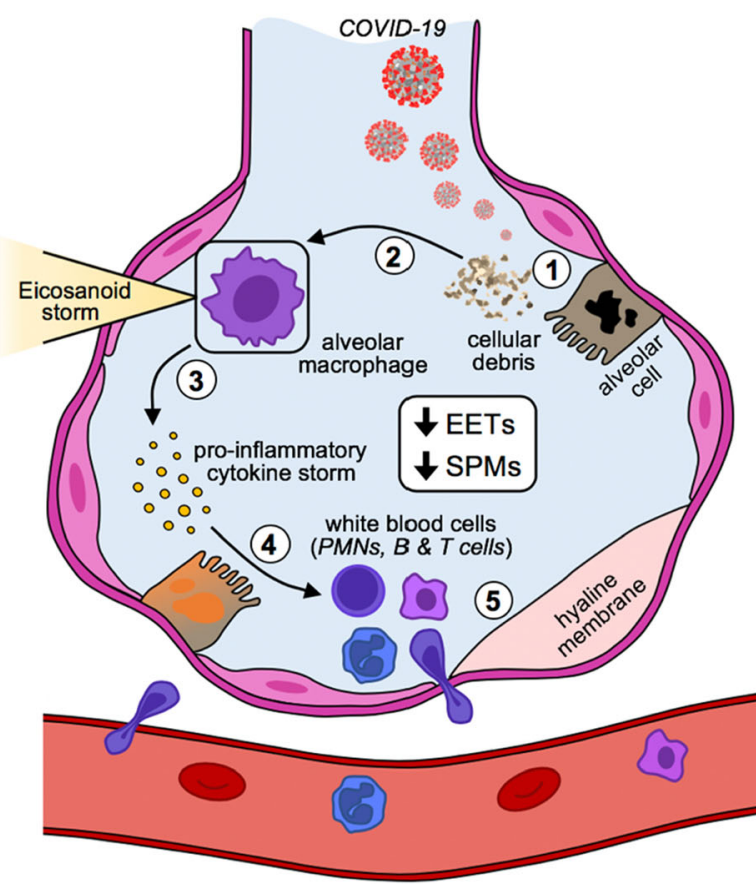

Fig. 1 Inflammation resolution in severe COVID-19 infection. The left alveolus demonstrates (1) viral-induced alveolar cell death and the accumulation of apoptotic and necrotic cellular debris. This debris elicits a proinflammatory response from macrophages (2), which in turn produce high quantities of eicosanoids generating an "eicosanoid storm". These eicosanoids subsequently stimulate the production of pro-inflammatory cytokines (3) by immune cells such as macrophages, generating a robust "cytokine storm" that in turn promotes further leukocytosis and immune cell infiltrates (4). This robust inflammatory response to viral infection promotes hyaline membrane formation (5) and subsequent acute respiratory endogenous resolution by stimulating macrophage phagocytosis of cellular debris and countering the release of proinflammatory cytokines/chemokines [5]. Importantly, loss of inflammation resolution mechanisms plays a role in sustaining pathologic inflammation [5]. Endogenous resolution processes have been identified in the termination of infectious diseases [5], including influenza [6-8], and could thus be harnessed for averting dysregulated inflammation and associated mortality in COVID-19. SPMs promote anti-viral B lymphocytic activity in influenza [7], suggesting they may be a promising therapy for COVID-19. SPM precursors including 17-hydroxydocosahexaenoic acid (17-HDHA) have also been identified as potentially promising vaccine adjuvants as they protect against primary influenza infection and promote adaptive immunity [7, 8]. Thus, the use of SPMs or their precursors in combination with COVID-19 vaccines may be a novel and effective therapeutic approach.

The resolution of inflammation is also stimulated by another pathway involving arachidonic acid-derived epoxyeicosatrienoic acids (EETs). These mediators promote clearance of cellular debris and activate anti-inflammatory programs to inhibit several

\section{INFLAMMATION-RESOLUTION}

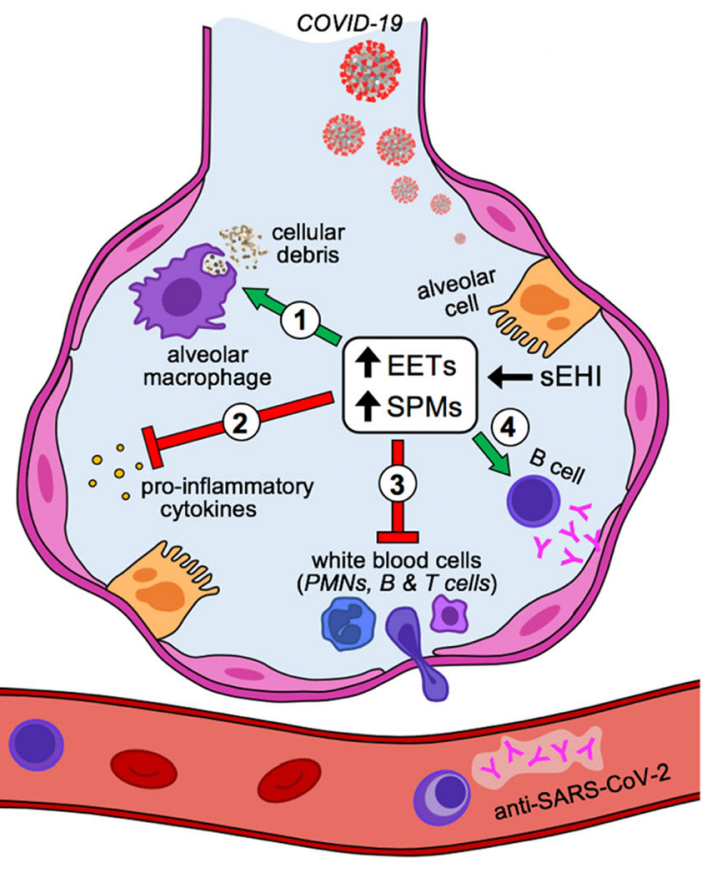

distress syndrome. Tissue levels of SPMs and EETs are decreased in this robust inflammatory setting. The right alveolus demonstrates COVID-19 infection in the setting of active inflammation resolution mechanisms, as is the case in mild infection or potentially following treatment with SPMs or sEH inhibition to increase EETs. EETs promote the generation of SPMs from arachidonic acid and omega-3 fatty acids. SPMs (1) stimulate macrophage phagocytosis and efferocytosis, (2) decrease pro-inflammatory cytokine production, (3) inhibit leukocytosis and thereby decrease the inflammatory infiltrate, and (4) may stimulate the adaptive immune response and the production of anti-SARS-CoV-2 antibodies 
key pro-inflammatory cytokines $[9,10]$. EETs and other epoxy fatty acids specifically promote production of SPMs, such as lipoxins, by shifting arachidonic acid metabolism to favor inflammation resolution [11]. As EETs are rapidly metabolized by soluble epoxide hydrolase (sEH), administration of sEH inhibitors (sEHIs) can stabilize EET levels, prevent lung inflammation, and improve lung function in animal models, making them an attractive potential therapeutic strategy for COVID-19. Both SPMs and SEHIs downregulate the transcription regulator $\mathrm{NF}-\mathrm{kB}[5,11]$, the center of eicosanoid-induced cytokine storms, which promotes the induction of pro-inflammatory cytokines and prostaglandin synthesis via cyclooxygenase (COX). Combined pharmacological abrogation of COX-2 and sEH activity also prevents cytokine and eicosanoid storms via debris clearance mechanisms [4], offering another promising therapeutic intervention.

Targeting individual pro-inflammatory cytokines may not be sufficient to prevent COVID-19 progression. Importantly, SPMs terminate self-sustaining inflammatory processes, such as those induced by COVID-19, by broadly inhibiting proinflammatory cytokine production and promoting a return to tissue homeostasis $[5,7,8]$. In contrast to some antiinflammatory agents, which may be contraindicated in COVID-19 patients, SPMs and sEHIs act at significantly lower doses and are not immunosuppressive [5, 11]. Moreover, conventional anti-inflammatory agents such as NSAIDs and COX-2 inhibitors, while limiting the eicosanoid storm, may be "resolution toxic" as they indiscriminately inhibit eicosanoid pathways that produce resolution mediators and thereby prevent active resolution [5]. Inhibiting resolution mediator production may potentially facilitate COVID-19-induced tissue injury and progression of infection. As demonstrated in many inflammatory disease models, selectively promoting endogenous inflammation resolution mechanisms clears inflammatory exudates more effectively and promotes a return to tissue homeostasis compared with classic anti-inflammatory agents [5-11].

Most COVID-19 clinical trials focus on "anti-viral" and "anti-inflammatory" strategies. In contrast, stimulation of inflammation resolution is a novel host-focused alternative to complement current therapies. The loss of active resolution mechanisms [5] and dysregulation of SPMs and EETs in sustained pathological inflammation and lung diseases present a new opportunity for the management of COVID-19. Pro-resolving mediators, as well as sEH blockade, may resolve the potentially fatal cytokine storm and severe pulmonary inflammation in COVID-19 patients (see Fig. 1). Importantly, both SPMs and EETs have also been shown to attenuate pathologic thrombosis and promote clot removal, which is now recognized as a key pathology of COVID-19 infection [12-14]. SPMs including resolvins, lipoxins, and protectins, as well as sEHIs, are currently in clinical trials for other inflammatory diseases and could be rapidly translated for the management of COVID-19 by complementing current anti-viral strategies via debris clearance and inflammatory cytokine suppression. Thus, activating endogenous resolution pathways may be a novel therapeutic approach to limit severe organ damage and improve outcomes in COVID-19 patients.

Acknowledgements The corresponding authors are supported by $\mathrm{NIH}$ grants including R01GM038765 (to CNS); National Institute of Environmental Health Science Superfund Research Program grant P42 ES004699, and National Institute of Environmental Health Science (River Award R35ES030443) (to BDH); and the Credit Unions Kids at Heart Team (to DP); the C.J. Buckley Pediatric Brain Tumor Fund (DP); and the Joe Andruzzi Foundation (to DP).

\section{References}

1. Mehta, P., McAuley, D. F., Brown, M., et al. (2020). COVID-19: consider cytokine storm syndromes and immunosuppression. Lancet, 395(10229), 1033-1034.

2. Fung, S. Y., Yuen, K. S., Ye, Z. W., Chan, C. P., \& Jin, D. Y. (2020). A tug-of-war between severe acute respiratory syndrome coronavirus 2 and host antiviral defence: lessons from other pathogenic viruses. Emerging Microbes \& Infections, 9(1), 558-570.

3. von Moltke, J., Trinidad, N. J., Moayeri, M., Kintzer, A. F., Wang, S. B., van Rooijen, N., Brown, C. R., Krantz, B. A., Leppla, S. H., Gronert, K., \& Vance, R. E. (2012). Rapid induction of inflammatory lipid mediators by the inflammasome in vivo. Nature, 490(7418), 107-111.

4. Gartung, A., Yang, J., Sukhatme, V. P., Bielenberg, D. R., Fernandes, D., Chang, J., Schmidt, B. A., Hwang, S. H., Zurakowski, D., Huang, S., Kieran, M. W., Hammock, B. D., \& Panigrahy, D. (2019). Suppression of chemotherapy-induced cytokine/lipid mediator surge and ovarian cancer by a dual COX$2 / \mathrm{sEH}$ inhibitor. Proceedings of the National Academy of Sciences of the United States of America, 116(5), 1698-1703.

5. Serhan, C. N. (2014). Pro-resolving lipid mediators are leads for resolution physiology. Nature, 510(7503), 92-101.

6. Tam, V. C., Quehenberger, O., Oshansky, C. M., Suen, R., Armando, A. M., Treuting, P. M., Thomas, P. G., Dennis, E. A., \& Aderem, A. (2013). Lipidomic profiling of influenza infection identifies mediators that induce and resolve inflammation. Cell, 154(1), 213-227.

7. Ramon, S., Baker, S. F., Sahler, J. M., Kim, N., Feldsott, E. A., Serhan, C. N., Martínez-Sobrido, L., Topham, D. J., \& Phipps, R. P. (2014). The specialized proresolving mediator 17-HDHA enhances the antibody-mediated immune response against influenza virus: a new class of adjuvant? Journal of Immunology, 193(12), 60316040.

8. Morita, M., Kuba, K., Ichikawa, A., Nakayama, M., Katahira, J., Iwamoto, R., Watanebe, T., Sakabe, S., Daidoji, T., Nakamura, S., Kadowaki, A., Ohto, T., Nakanishi, H., Taguchi, R., Nakaya, T., Murakami, M., Yoneda, Y., Arai, H., Kawaoka, Y., Penninger, J. M., Arita, M., \& Imai, Y. (2013). The lipid mediator protectin D1 inhibits influenza virus replication and improves severe influenza. Cell, 153(1), 112-125.

9. Gilroy, D. W., Edin, M. L., De Maeyer, R. P., et al. (2016). CYP450-derived oxylipins mediate inflammatory resolution. Proceedings of the National Academy of Sciences of the United States of America, 113(23), E3240-E3249.

10. Node K, Huo Y, Ruan X, et al. (1999). Anti-inflammatory properties of cytochrome P450 epoxygenase-derived eicosanoids. Science, 285(5431), 1276-9. 
11. Schmelzer, K. R., Kubala, L., Newman, J. W., Kim, I. H., Eiserich, J. P., \& Hammock, B. D. (2005). Soluble epoxide hydrolase is a therapeutic target for acute inflammation. Proceedings of the National Academy of Sciences of the United States of America, 102(28), 9772-9777.

12. Cherpokova, D., Jouvene, C. C., Libreros, S., DeRoo, E. P., Chu, L., de la Rosa, X., Norris, P. C., Wagner, D. D., \& Serh, C. N. (2019). Resolvin D4 attenuates the severity of pathological thrombosis in mice. Blood 134 (17), 1458-1468.
13. Imig JD, Hammock BD. (2009). Soluble epoxide hydrolase as a therapeutic target for cardiovascular diseases. Nature Reviews Drug Discovery, 8(10), 794-805.

14. Zhang Y, Xiao M, Zhang S, et al. (2020). Coagulopathy and Antiphospholipid Antibodies in Patients with Covid-19. The New England Journal of Medicine, 382(17), e38.

Publisher's note Springer Nature remains neutral with regard to jurisdictional claims in published maps and institutional affiliations. 